\title{
Exceptional high-strain-rate tensile mechanical properties in a CrCoNi medium-entropy alloy
}

\author{
Peng $\mathrm{Gao}^{1 \dagger}$, Zihao $\mathrm{Ma}^{3 \dagger}$, Ji Gu${ }^{2}$, Song $\mathrm{Ni}^{2}$, Tao Suo ${ }^{3}$, Yulong $\mathrm{Li}^{3^{*}}$, Min Song ${ }^{2 *}$, Yiu-Wing $\mathrm{Mai}^{1}$ and \\ Xiaozhou Liao ${ }^{1^{*}}$
}

\begin{abstract}
Alloys with combined outstanding strength and excellent ductility are highly desirable for many structural applications. However, alloys subjected to deformation at very high strain rates and/or cryogenic temperatures usually suffer from very limited ductility. Here, we demonstrate that a bulk CrCoNi medium-entropy alloy presents exceptional combination of high strength and excellent ductility during deformation at high strain rates over a wide temperature range. Full tensile stress-strain curves at a high strain rate of $2000 \mathrm{~s}^{-1}$ and temperatures down to $77 \mathrm{~K}$ were successfully obtained using an electromagnetic Hopkinson tension bar system attached with a cooling device, revealing high true ultimate tensile strength $\left(\sigma_{\mathrm{UTS}, \mathrm{T}}\right)$ of $1.8 \mathrm{GPa}$ and true strain of $\sim 54 \%$ at $\sigma_{\text {UTS,T. }}$ These outstanding mechanical properties were mainly attributed to profuse deformation twinning. Both high strain rate and cryogenic temperature promoted deformation twinning. Grain refinement caused by deformation twinning, dislocation slip and dynamic recrystallisation added to work hardening and the excellent tensile strain.
\end{abstract}

Keywords: medium-entropy alloy, strength, ductility, high strain rate, cryogenic temperature

\section{INTRODUCTION}

Metallic materials with combined outstanding strength and excellent ductility are highly desirable for many applications. However, most metallic materials working under cryogenic temperatures and/or high strain rates suffer from poor ductility. Low temperature makes dislocation slip difficult, which normally results in decreased ductility in most engineering materials [1-3]. High strain rate does not provide enough time for slip, leading to localised dislocation concentration and consequently poor ductility [4]. Recently, there have been reports that some high/medium-entropy alloys (H/MEAs) present superior strength and excellent ductility at cryogenic temperatures [5-7]. The key reason that contributes to the outstanding mechanical properties at cryogenic temperatures is extensive deformation twinning activities, which strengthen materials and facilitate plastic deformation $[5,6,8]$.

It is well-known that the propensity of deformation twinning is remarkably influenced by factors including stacking fault energy, deformation temperature and strain rate [9]. Lowering stacking fault energy, which can be achieved by changing the composition of alloys, promotes deformation twinning [9]. It has been reported that reducing temperature also lowers stacking fault energy and thus promotes deformation twinning [10]. Another explanation on the temperature effect on deformation twinning is that it usually occurs at relatively high stresses and low temperature increases the flow stress and therefore facilitates twinning [11]. It is believed that increasing the deformation strain rate has a similar effect as decreasing the temperature in facilitating deformation twinning [12]. Given that some $\mathrm{H} /$ MEAs have exceptional strength and ductility at cryogenic temperatures because of pronounced deformation twinning, it is reasonable to expect that they would also yield combined high strength and excellent ductility during high-strain-rate deformation. However, there has been no report on the high-strainrate tensile mechanical properties of $\mathrm{H} / \mathrm{MEAs}$ because of the difficulty in acquiring complete high-strain-rate tensile stressstrain curves of materials with high ductility. The available literature related to high-strain-rate deformation of H/MEAs failed to present complete stress-strain curves due to the remarkable ductility and strength that the H/MEAs can reach under dynamic deformation [13]. Other than studies done under dynamic conditions, the combined effects of cryogenic temperature and high strain rate on the tensile mechanical properties of H/MEAs have never been reported.

Herein, we used a home-made electromagnetic split Hopkinson tension bar (SHTB) system with a cooling device to successfully conduct high-strain-rate tensile deformation of a CrCoNi MEA until failure. The deformation strain rate was $2000 \mathrm{~s}^{-1}$ and the deformation was conducted at a wide temperature range between $298 \mathrm{~K}$ (room temperature, RT) and $77 \mathrm{~K}$. Results revealed exceptional combined true ultimate tensile strength $\left(\sigma_{\mathrm{UTS}, \mathrm{T}}\right)$ and ductility at high strain rates in this temperature range. High propensity of deformation twinning activities and significant grain refinement contributed to the exceptional mechanical properties.

\section{EXPERIMENTAL SECTION}

Materials processing and quasi-static tests

Raw CrCoNi ingots with dimensions of $150 \mathrm{~mm} \times 60 \mathrm{~mm} \times$

\footnotetext{
${ }^{1}$ School of Aerospace, Mechanical \& Mechatronic Engineering, The University of Sydney, Sydney, NSW 2006, Australia

${ }^{2}$ State Key Laboratory of Powder Metallurgy, Central South University, Changsha 410083, China

${ }^{3}$ School of Aeronautics, Northwestern Polytechnical University, Xi'an 710072, China

$\dagger$ These authors contributed equally to this work.

*Corresponding authors (emails: msong@csu.edu.cn (Song M); liyulong@nwpu.edu.cn (Li Y); xiaozhou.liao@sydney.edu.au (Liao X))
} 
$40 \mathrm{~mm}$ were prepared by vacuum induction melting in a highpurity argon atmosphere. The ingots were then homogenised at $1273 \mathrm{~K}$ for $2 \mathrm{~h}$, followed by hot rolling with thickness reduction of $\sim 50 \%$ at $973 \mathrm{~K}$. The dimensions of the final hot-rolled plates were about $270 \mathrm{~mm} \times 65 \mathrm{~mm} \times 20 \mathrm{~mm}$. Dog-bone samples for tension were cut from the hot-rolled plates. A schematic of the sample dimensions is shown in Fig. S1. Quasi-static (QS) tensile tests at RT were conducted using a CSS-44100 electronic motorcontrolled tensile testing machine at a strain rate of $10^{-3} \mathrm{~s}^{-1}$. Three samples were tested for all mechanical tests.

\section{High-strain-rate tensile tests}

A symmetric electromagnetic SHTB equipped with a cooling case was successfully used to acquire the complete high-strainrate tensile stress-strain curves at different temperatures. Engineering strain in QS tests was obtained from the displacement of a CSS-44100 electronic motor-controlled tensile testing machine. Engineering strain in SHTB tests was acquired using strain sensors that were bonded on the incident bars. Engineering failure strain was confirmed by manually measuring the total length of fractured samples. A schematic illustration of the symmetric electromagnetic SHTB is given in Fig. S2. To achieve a stress pulse long enough to fracture a specimen, two electromagnetic SHTBs were connected in parallel to form a symmetrical electromagnetic SHTB. The stress pulse of the electromagnetic SHTB was produced by the electromagnetic force and, therefore, the stress pulse that loaded the specimen could be accurately controlled [14]. Dog-bone samples were thread-fixed between the two incident bars and symmetrical loading was applied by an electromagnetic force through discharging the capacitor bank. The maximum strain of the specimen could reach twice that of a traditional mechanical SHTB and the specimen was always in an equilibrium condition compared to the conventional Hopkinson system. Details of the instrumental setup and working mechanism of the electromagnetic Hopkinson tension bar system are given in a previous study [14]. Dynamic tensile experiments were conducted at a strain rate of $2000 \mathrm{~s}^{-1}$ and temperatures of RT, 200 and $77 \mathrm{~K}$. The length of the two incident bars was $3.5 \mathrm{~m}$. A foam case was used to house the sample and control the temperature using liquid $\mathrm{N}_{2}$. A thermometer was inserted into the case and positioned close to the sample. Three samples were tested for each deformation condition and all samples were pulled to fracture.

\section{Sample preparation and experimental details for electron back- scattered diffraction \\ Samples for electron back-scattered diffraction (EBSD) were fixed in conductive resin, mechanically ground using $\mathrm{SiC}$ papers and then polished by a $0.25-\mu \mathrm{m}$ silica suspension. EBSD char- acterisation was conducted using a Zeiss Ultra field emission scanning electron microscope working at $20 \mathrm{kV}$. The scanning step sizes were 1 and $0.05 \mu \mathrm{m}$ for samples before and after deformation, respectively. The microstructural data were recorded by Aztec and analysed using the Channel 5 software (HKL Technology) to acquire detailed information on grain orientation, boundary characteristics and the density of geo- metrically necessary dislocations (GNDs).}

Experimental details for transmission Kikuchi diffraction and transmission electron microscopy

Specimens for transmission Kikuchi diffraction (TKD) and transmission electron microscopy (TEM) characterisation were prepared using the focused ion beam (FIB) technique in a Helios G4 Plasma FIB. The FIB voltage and current of $30 \mathrm{kV}$ and $100 \mathrm{pA}$, and $5 \mathrm{kV}$ and $100 \mathrm{pA}$ were used for the milling and final polishing, respectively. TKD was conducted in a Zeiss ULTRA Plus scanning electron microscope operating at $30 \mathrm{kV}$ (step size $6.5 \mathrm{~nm}$ ) and TEM in a JEOL 2200FS operating at $200 \mathrm{kV}$.

\section{RESULTS}

Yield strength, $\sigma_{\mathrm{UTS}, \mathrm{T}}$, and true strain at $\sigma_{\mathrm{UTS}, \mathrm{T}}$

Dog-bone samples were tested to obtain full stress-strain curves at a high uniaxial tensile strain rate. Typical engineering and true stress-strain curves are shown in Fig. 1a but the discussion below focuses on the true stress-strain curves. Full engineering stress-strain curves were recorded and the true stress-strain data were obtained from engineering stress-strain curves. Here, the true stress-strain data before the occurrence of obvious necking were presented. Also, $\sigma_{\mathrm{UTS}, \mathrm{T}}$ was calculated from the engineering ultimate tensile strength $\left(\sigma_{\mathrm{UTS}, \mathrm{E}}\right)$. QS tensile tests were only conducted at RT and the results are also included in Fig. 1a for comparison. Insets in the lower left and right corners of Fig. 1a show the samples before and after deformation for visual comparison. All the samples were pulled to failure and their mechanical properties are listed in Table 1. Both yield strength $\left(0.2 \%\right.$ offset yield strength) and $\sigma_{\mathrm{UTS}, \mathrm{T}}$ increased significantly with strain rate. For deformation at a high strain rate of $2000 \mathrm{~s}^{-1}$, lowering the temperature from RT to 200 and $77 \mathrm{~K}$ further increased $\sigma_{\text {UTS,T. }}$ Strengths increased dramatically with increasing strain rate. The true strain at $\sigma_{\mathrm{UTS}, \mathrm{T}}$, which gives a smaller value than the value of ductility (engineering tensile plastic strain at failure), was used to represent the ductility in this study, and was compared with the true strain at failure of other materials. The influence of strain rate on strength and ductility was more significant than that of temperature. The true stressstrain curves at different temperatures and $2000 \mathrm{~s}^{-1}$ almost overlapped to each other in the first half of the total deformation. However, in the second half of the deformation, low temperatures ( 200 and $77 \mathrm{~K}$ ) further increased the true stress. Note that previous reports demonstrated significant impacts of low temperature on the strength and ductility of similar $\mathrm{H}$ / MEAs at QS tensile tests because of significant deformation twinning at low temperatures [8]. These impacts were obscured in high-strain-rate tensile experiments because of the predominant effect of high strain rate on deformation twinning over that at low temperatures. As such, the mechanical properties were less affected by lowering temperature in high-strainrate deformation. At a strain rate of $2000 \mathrm{~s}^{-1}$, work hardening in the late stages of deformation was enhanced with decreasing temperature, as shown in Fig. 1b.

\section{Comparison of mechanical properties with other alloys}

Fig. 1c compares the mechanical properties of our samples and other alloys, including other H/MEAs, twinning-induced plasticity (TWIP) steels, Ti alloys and light alloys, deformed at high strain rates and low temperatures. Due to the limited dynamic tensile test data available in the literature, data from dynamic compression tests are also included here. Red symbols represent data from the present study. Green and black symbols represent tensile and compression data, respectively, from other studies. To the best of our knowledge, our CrCoNi samples show the 

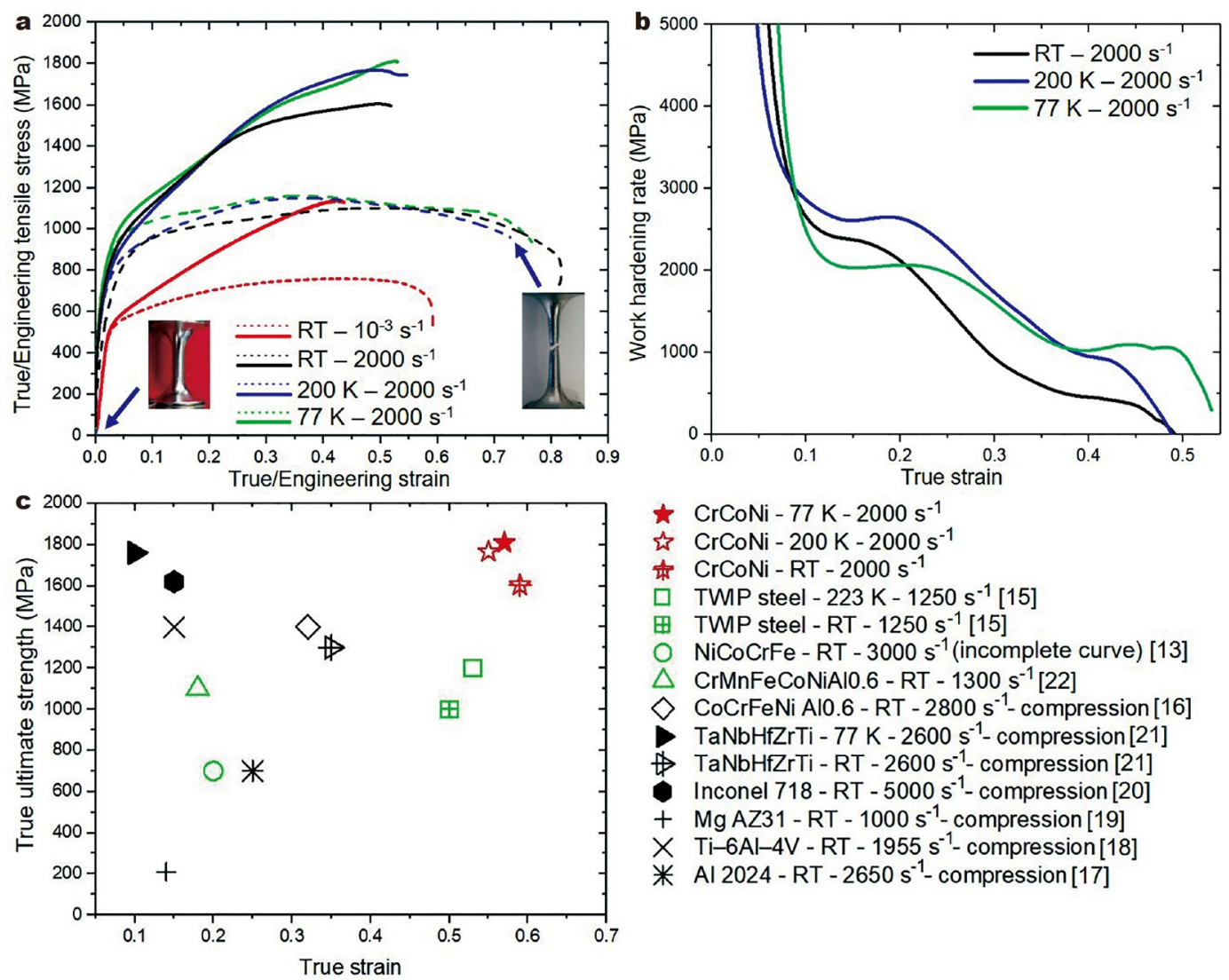

Figure 1 Mechanical properties and work hardening rates of CrCoNi samples. (a) True (solid lines) and engineering (dashed lines) tensile stress-strain curves of $\mathrm{CrCoNi}$ samples at QS and a high strain rate at RT and cryogenic temperatures. Insets at the lower left and right corners show sample photos (at the same scale) before tensile loading and at failure, respectively. (b) Work hardening rates of samples. (c) Comparison of true ultimate strengths of CrCoNi and other engineering alloys. Red symbols indicate $\mathrm{CrCoNi}$ data from the present study. Green symbols represent tensile test data and black symbols represent compression tests from other studies [13,15-22].

Table 1 Mechanical properties, including yield strength, true ultimate tensile strength $\left(\sigma_{\mathrm{UTS}, \mathrm{T}}\right)$ and true strain at $\sigma_{\mathrm{UTS}, \mathrm{T}}$, of the bulk CrCoNi alloy at various strain rates and temperatures

\begin{tabular}{ccccc}
\hline Strain rate $\left(\mathrm{s}^{-1}\right)$ & Temperature $(\mathrm{K})$ & Yield strength $(\mathrm{MPa})$ & $\sigma_{\text {UTS,T }}(\mathrm{MPa})$ & True strain at $\sigma_{\text {UTS,T }}(\%)$ \\
\hline $10^{-3}(\mathrm{QS})$ & $\mathrm{RT}$ & $509 \pm 36$ & $1130 \pm 9$ & $43 \pm 1$ \\
2000 & $\mathrm{RT}$ & $805 \pm 41$ & $1603 \pm 62$ & $53 \pm 1$ \\
2000 & 200 & $772 \pm 58$ & $1766 \pm 45$ & $55 \pm 6$ \\
2000 & 77 & $872 \pm 59$ & $1809 \pm 50$ & $54 \pm 2$ \\
\hline
\end{tabular}

best overall dynamic strength and ductility at RT and cryogenic temperatures among engineering materials reported so far [13,15-22]. TWIP steels also exhibited excellent ductility at cryogenic temperatures, attributed to deformation twinning [15]. CrFeCoNi H/MEA was tested at $3000 \mathrm{~s}^{-1}$; however, complete curves were not obtained using a traditional SHTB system [13]. The TiZrNbHfTa HEA presented very high strength with very limited strain [21], probably due to its body-centred cubic structure possessing fewer slip systems. Inconel 718 and Ti-6Al$4 \mathrm{~V}$ exhibited high true ultimate compressive strength values, while their true compressive strain at failure were also relatively low $[18,20]$.

\section{Microstructure}

Fig. 2 displays the microstructures of the samples before and after deformation. Fig. 2a presents an image of a fully fractured sample with two areas marked as "Area 1" and "Area 2". Area 1 was $\sim 2 \mathrm{~mm}$ away from the fracture surface, which was between the end of the homogeneous deformation and the start of neck. The microstructure in Area 1 largely reflected that of the homogeneous deformation region. Area 2 was immediately adjacent to the fracture surface. The local strain in Area 1 was similar to the strain at incipient necking, i.e., almost the same as the true strain at $\sigma_{\mathrm{UTS}, \mathrm{T}}, 55 \%$. As non-uniform deformation occurred in the necking region, it is more appropriate to use percent reduction in area (\%RA) to quantify local strain in the region. The \%RA in Area 2 was $70 \%$. For comparison, the \%RA in Area 1 was $36 \%$. Fig. 2b shows a typical EBSD inverse pole figure (IPF) map and corresponding $\{100\},\{110\}$ and $\{111\}$ pole figures of microstructure before deformation. Grains in the material presented random orientation with an average grain size of $\sim 26 \mu \mathrm{m}$. There were many coarse annealing twins with 

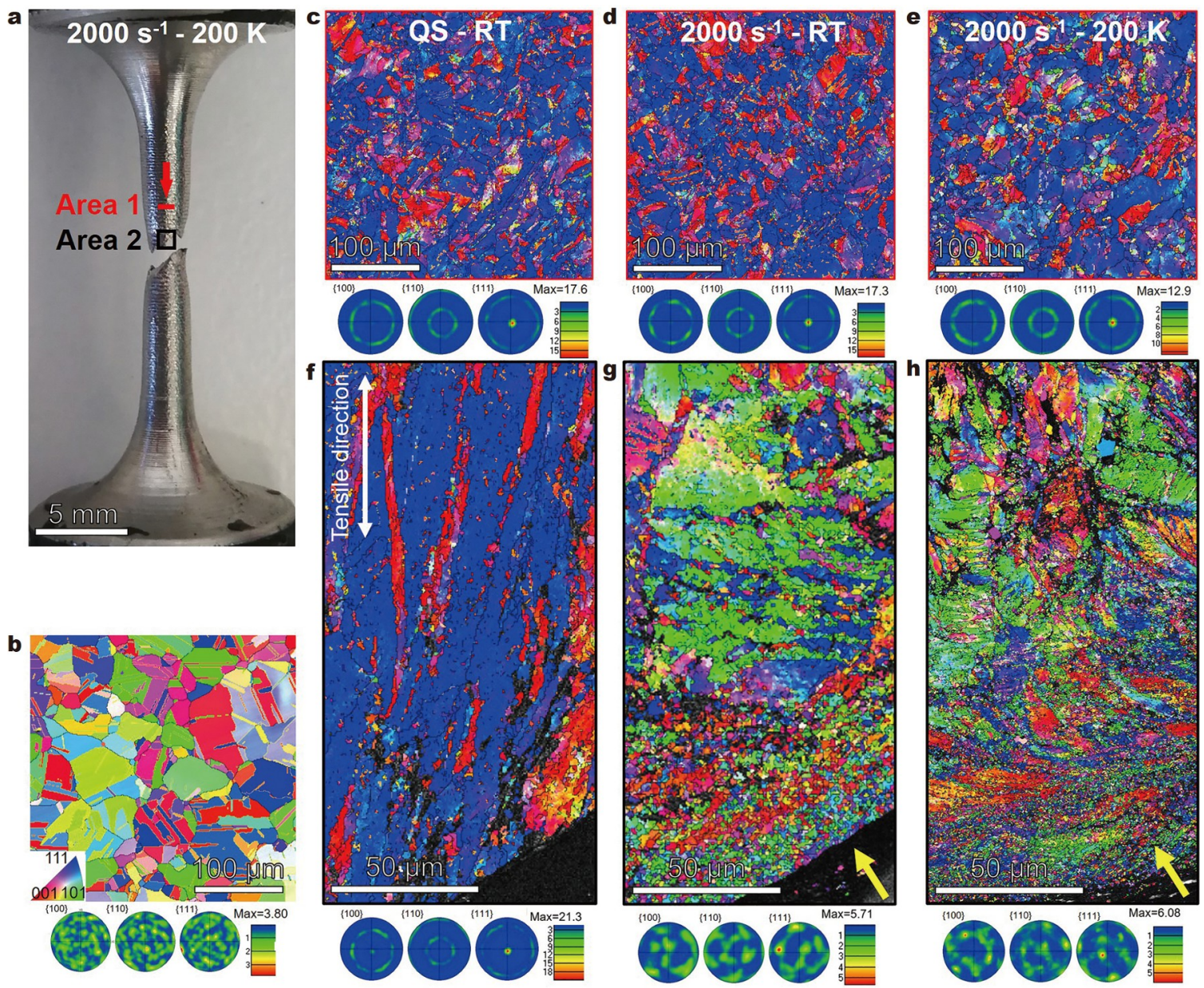

Figure 2 Microstructures of samples before and after deformation observed via EBSD. (a) An example (2000 s ${ }^{-1}$ at $\left.200 \mathrm{~K}\right)$ of a fractured sample after highstrain-rate tensile deformation showing sampling areas. (b) EBSD IPF map and pole figures before deformation. EBSD IPF map and pole figures of Area 1 after (c) QS at RT, (d) $2000 \mathrm{~s}^{-1}$ at RT, and (e) $2000 \mathrm{~s}^{-1}$ at $200 \mathrm{~K}$. EBSD IPF map and pole figures of Area 2 after (f) QS at RT, (g) $2000 \mathrm{~s}^{-1}$ at RT, and (h) $2000 \mathrm{~s}^{-1}$ at $200 \mathrm{~K}$. The tensile direction is perpendicular to the image planes of $(\mathrm{c}-\mathrm{e})$. The tensile direction marked in (f) applies to (f-h). The colour code at the lower left corner of (b) applies to all EBSD maps in Figs 2 and 3.

widths and twin spacings of $\sim 5-10$ and $\sim 10-20 \mu \mathrm{m}$, respectively. Most of the twins did not pass through a whole grain and therefore the lengths were in the range of $\sim 10-20 \mu \mathrm{m}$. After deformation, strong texture developed in Area 1 in all samples, as displayed in the IPFs and pole figures in Fig. 2c-e. Because the mechanical properties and microstructures at 200 and $77 \mathrm{~K}$ were very similar, only the microstructures of samples deformed at $200 \mathrm{~K}$ are presented here. It is common for face-centered cubic alloys to have a dominant $\langle 111\rangle$ and minor $\langle 100\rangle$ texture along the tensile direction after uniaxial tensile deformation [23,24]. The normal directions of the $\{111\}$ slip planes of grains with different crystallographic orientations are inclined to rotate towards the tensile direction. When the tensile direction is perpendicular to the $\{111\}$ slip plane, the resolved shear stress on the plane approaches zero [25], so that dislocation slip on the plane stops and grain rotation also stops. The $\langle 100\rangle$ texture stems from deformation twinning [23]. The average grain sizes in Fig. 2c-e were 6.5, 6.4 and $6.1 \mu \mathrm{m}$, respectively, which did not differ much. The microstructures of large areas from samples dynamically deformed at RT and $200 \mathrm{~K}$ are shown in Fig. S3, indicating more deformation twins at $200 \mathrm{~K}$ than at RT.

The microstructures in Area 2 of the samples are shown in
Fig. 2f (QS deformation at RT), 2g (dynamic deformation at $\mathrm{RT}$ ), and $2 \mathrm{~h}$ (dynamic deformation at $200 \mathrm{~K}$ ). Because the samples in Area 2 experienced large plastic deformation, some sample areas were of very fine grain sizes and/or very high densities of crystalline defects and were hence not identified by EBSD. These areas appear as black areas in the figures. Fig. $2 \mathrm{f}$ shows that, after QS deformation, relatively coarse grains were stretched along the pulling direction. Fig. $2 \mathrm{~g}$, h display structures with some resemblance. Both show large amounts of ultrafine-/ nano-grains near the fracture surface, as indicated by the yellow arrows. The results show that the thickness of the region with ultra-fine/nano grains in samples deformed at RT and $200 \mathrm{~K}$ was $\sim 70$ and $\sim 100 \mu \mathrm{m}$, respectively. The thickness of ultrafine-/nanograins layers accounted for $\sim 2 \%(0.07 \mathrm{~mm} \times 2 / 6 \mathrm{~mm})$ of the engineering tensile strain at RT and $\sim 3 \%(0.1 \mathrm{~mm} \times 2 / 6 \mathrm{~mm})$ at $200 \mathrm{~K}$. Since the strain values of these areas are minor to the total strain values, only the micro-structures outside these areas will be discussed. The average grain size of the ultra-fine/nano grains area for samples deformed at RT and $200 \mathrm{~K}$ was 0.54 and $0.28 \mu \mathrm{m}$, respectively. The average grain size excluding the ultrafine-grained region shown in Fig. $2 \mathrm{f}-\mathrm{h}$ was $3.4,3.0$ and $1.2 \mu \mathrm{m}$, respectively. In both Fig. $2 \mathrm{~g}$, h, many grains with a $<110\rangle$ 
crystallographic orientation (green areas) were seen. Detailed microstructures of areas away from the ultrafine-grained regions will be shown later.

The TKD IPF maps in Fig. 3 show details of typical microstructures from the necking areas of the same three deformed samples as presented in Fig. 2. They were taken from regions that were $\sim 200 \mu \mathrm{m}$ from the fracture surfaces. High densities of primary and secondary twins were observed in all samples. Fig. 3a shows that under QS condition, secondary twins intersected with primary twins, as indicated by white circles at two places, which is believed to improve both the strength and ductility [26]. Fig. 3b shows the microstructure at $2000 \mathrm{~s}^{-1}$ and RT. Red arrows point to primary twins. Intense twin-twin interactions were also found here. Some twins, a few of which are indicated by red arrows, had a $<110>$ axis along the tensile direction. Fig. $3 c$ presents a magnified image of the area marked with the black box in Fig. 3b, showing that fine grains formed around twinned areas, as indicated by the black arrows. They displayed relatively random orientations, as suggested by the multiple pseudo-colours, indicating that they were dynamically recrystallised (DRX) grains [27]. Nano-sized sub-grains bounded by low-angle grain boundaries (LAGBs) were also seen, as indicated by the white arrow, which is an indication of intensive dislocation activities. Fig. 3d displays the microstructure at $2000 \mathrm{~s}^{-1}$ and $200 \mathrm{~K}$. The spacing and width of primary twin boundaries (TBs) were similar to those tested at $2000 \mathrm{~s}^{-1}$ and RT. However, the number of secondary TBs was dramatically larger. The density of TBs at cryogenic temperatures was higher than that at RT. Like the contribution of TBs to the superior ductility and strength of MEAs at cryogenic temperatures [5,8], TBs also contributed crucially to the exceptional mechanical properties of
CrCoNi observed at high strain rates. Many nano-grains formed around TBs, as indicated by the black arrows in Fig. 3e, a zoomin area in the white box of Fig. 3d. Nano-grains can form by either deformation-induced grain refinement or DRX. Deformation-induced grain refinement usually leads to nano-grains with relatively high dislocation densities within individual grains and, in many situations, neighbouring grains are separated by LAGBs [28]. In contrast, DRX grains are of very low dislocation densities (see Fig. 4a). Based on our structural characterization, some of the nano-grains observed here were very likely caused by DRX. This phenomenon is consistent with the report that TBs can act as preferred zones for DRX [29]. The formation of these nano-grains was very likely caused by DRX. The DRX led to low dislocation densities in addition to the relatively random grain orientations. Some recrystallised grains presented more random orientations, including $<110>$.

Statistical data on the spacings, widths and densities of the deformation twins in three samples are summarised in Table 2. Each data set was extracted from image areas of $5 \mu \mathrm{m} \times 5 \mu \mathrm{m}$ of typical twin areas of the samples. The twin densities were calculated based on the length of TBs in the observed areas. The density of TBs increased with increasing strain rate and/or reducing temperature.

Fig. 4 presents details of typical dynamic recrystallised grains in a sample at $200 \mathrm{~K}$. Fig. 4a shows some ultrafine-grained grains, two of which are marked with red dash loops, with very low dislocation densities. Meanwhile, nano-twins, some of which interact with each other, were seen within nano-grains (Fig. 4b). Stacking faults (SFs) were seen within nano-twins (Fig. 4c).

Twins contribute to the mechanical properties of materials because TBs act as barriers for dislocation motion, thus pre-
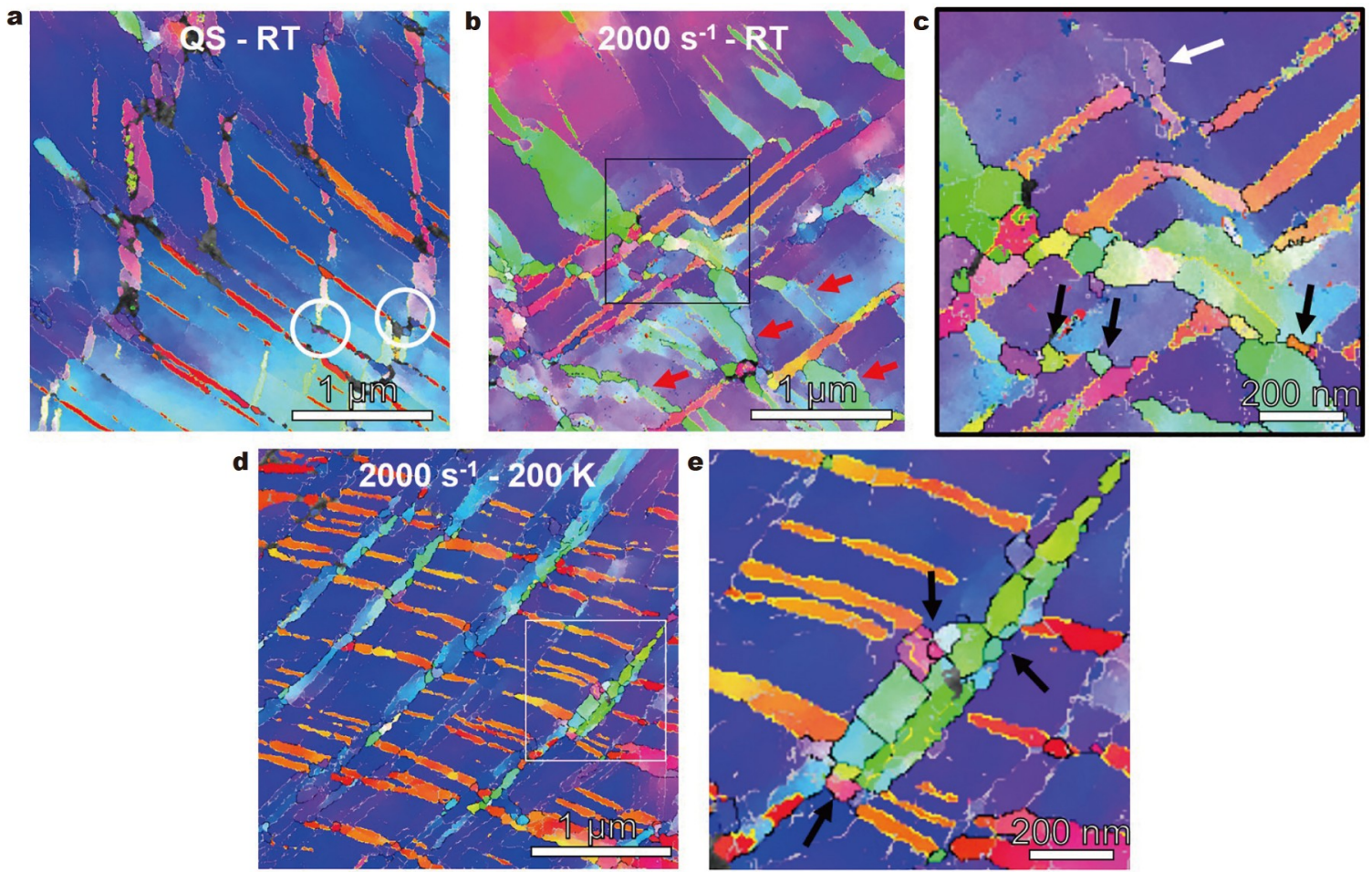

Figure 3 Detailed microstructures of fracture areas of deformed samples observed via TKD. TKD IPF maps of samples deformed at (a) QS, (b) $2000 \mathrm{~s}^{-1}$ at RT with a zoom-in image of the area marked with the black square presented in (c), and (d) $2000 \mathrm{~s}^{-1}$ at $200 \mathrm{~K}$ with a magnified image of the area marked with the white square presented in (e). White circles in (a) indicate that secondary twins blocked by primary twins. Red arrows in (b) point to primary twins. Black arrows in (c) and (e) point to DRX grains. The white arrow in (c) points to sub-grains formed by slip. 

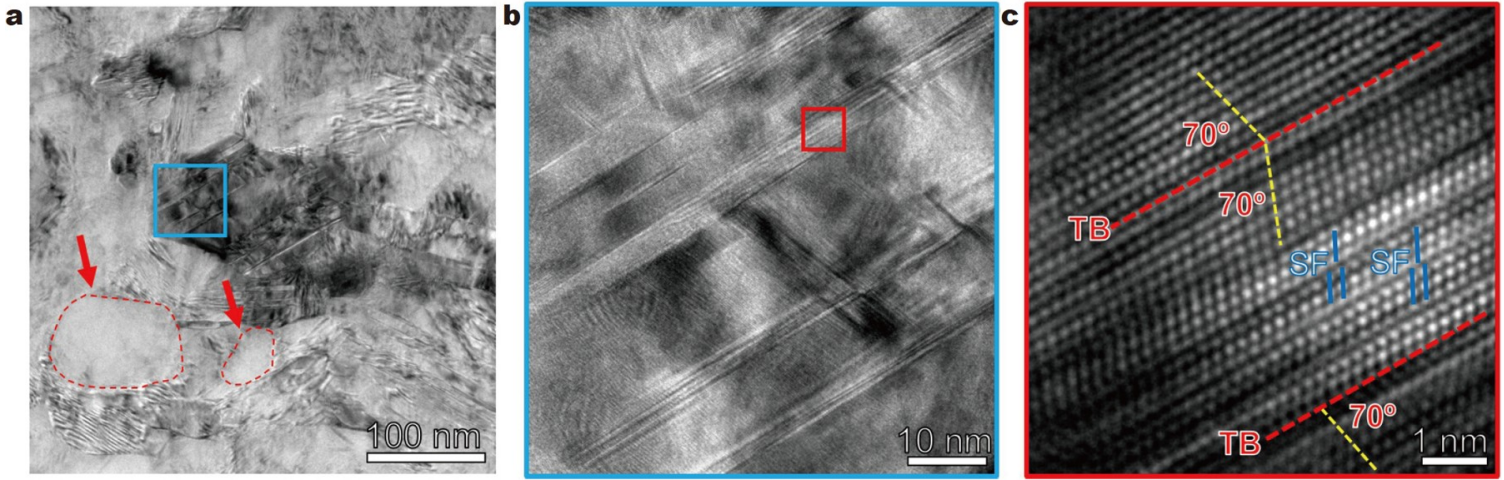

Figure 4 The microstructure of dynamic recrystallised grains of a sample dynamically deformed at $200 \mathrm{~K}$. (a) Nano grains near the fracture surface. Two grains with almost no dislocation are marked with red dash loops and indicated by red arrows. (b) An enlarged image of the area marked by the blue square in (a). (c) An enlarged image of the area marked by the red square in (b). TBs and SFs in (c) are indicated.

Table 2 Spacings, widths and densities of twins of deformed samples

\begin{tabular}{cccccc}
\hline \multirow{2}{*}{$\begin{array}{c}\text { Test parameters (strain rate- } \\
\text { temperature) }\end{array}$} & \multicolumn{2}{c}{ Primary twins } & \multicolumn{2}{c}{ Secondary twins } & \multirow{2}{*}{ TB density $\left(\mathrm{nm}{ }^{-1}\right)$} \\
\cline { 2 - 5 } & Spacing $(\mathrm{nm})$ & Width $(\mathrm{nm})$ & Spacing $(\mathrm{nm})$ & Width $(\mathrm{nm})$ & $50-100$ \\
QS-RT & $700-1200$ & $50-120$ & $100-580$ & $30-150$ & $5.9 \times 10^{-3}$ \\
2000 s $^{-1}-\mathrm{RT}$ & $300-500$ & $50-150$ & $150-800$ & $20-100$ & $8.7 \times 10^{-3}$ \\
$2000 \mathrm{~s}^{-1}-200 \mathrm{~K}$ & $300-1000$ & $80-150$ & $20-200$ & 2 \\
\hline
\end{tabular}
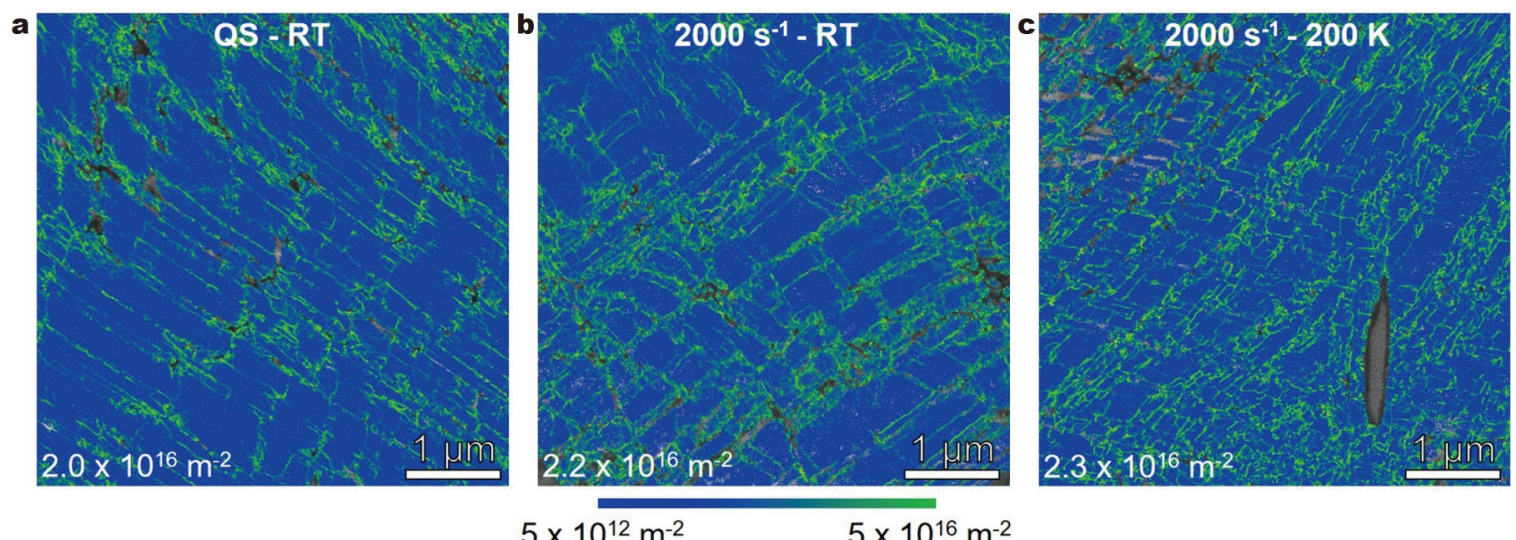

$$
5 \times 10^{12} \mathrm{~m}^{-2} \quad 5 \times 10^{16} \mathrm{~m}^{-2}
$$

Figure 5 The density maps of GNDs for deformed samples at (a) QS at RT, (b) $2000 \mathrm{~s}^{-1}$ at RT, and (c) $2000 \mathrm{~s}^{-1}$ at $200 \mathrm{~K}$. The colour legend is for the density of GNDs.

venting high local dislocation concentration during dynamic deformation, and effectively increasing the dislocation storage capability of the materials [30]. It is critical to understand how strain rate and temperature influence the density and distribution of dislocations in deformed samples. The density and distribution of GNDs, which contribute to the variation in local lattice orientation, can be visualised using TKD [31-33]. The results are presented in Fig. 5. The colour legend is for the GND density maps. The dark grey areas were not identified by TKD. For samples deformed at RT, with increasing strain rate, TBs hindered or localised more GNDs, displayed as green belts in Fig. 5a, b. At $200 \mathrm{~K}$, GNDs were more evenly distributed because TBs increased and were evenly distributed, as shown in Fig. 5c. The density of GNDs increased with increasing strain rate and decreasing temperature, which matches very well the variation of the TB density in these samples. In addition to the difference in average density of GNDs, the distributions of GNDs varied according to TBs.

\section{DISCUSSION}

\section{Deformation mechanisms}

The microstructures in Area 1 of all samples were very similar (Fig. 2c-e). Variation in microstructural evolution mainly occurred in Area 2. Fig. 6 presents schematic diagrams of deformation-induced microstructural evolutions under QS and dynamic deformation based on the experimental observations shown in Figs 2-4. Although the as-received samples contained many annealing twins, these twins were significantly larger than the twins shown in the deformed samples, implying that the latter were deformation twins formed during the plastic deformation process. Hence, the influence of annealing twins on 


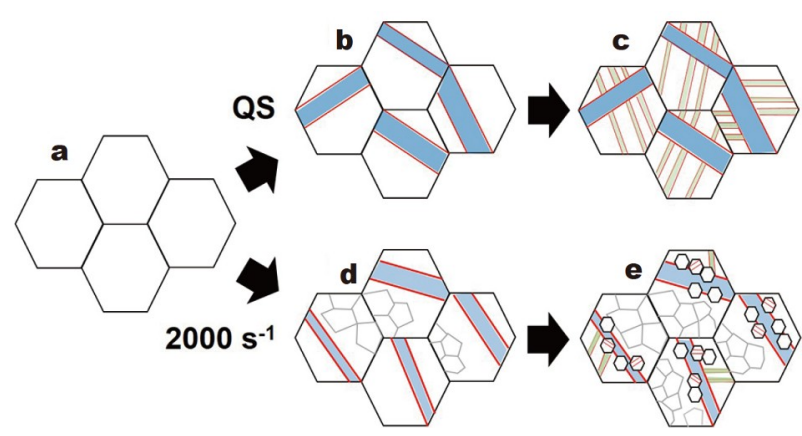

Figure 6 Schematics of the microstructural evolution processes during QS and dynamic deformation. (a) Microstructure before deformation. (b) Microstructure at early deformation stages under QS deformation. (c) Microstructure at late deformation stages under QS deformation. (d) Microstructure at early deformation stages under dynamic deformation. (e) Microstructure at late deformation stages under dynamic deformation. Images are not to scale. Black, red and grey lines represent conventional high-angle GBs, TBs and LAGBs, respectively. Blue and yellow areas represent primary and secondary twins, respectively.

microstructural evolution is not discussed.

Fig. 6 shows schematics of the microstructural evolution processes during QS and dynamic deformation. Before deformation, the material comprises equiaxed grains (Fig. 6a). For samples experiencing QS deformation at RT, primary twinning occurs (Fig. 6b), producing twins (in blue) with relatively large lengths and widths. A high density of secondary twins (in yellow) subsequently forms. But most of the secondary twins are blocked by the primary twins (Fig. 6c). For samples experiencing dynamic deformation, many primary and secondary twins form with increased densities, i.e., high strain rate promotes twinning. Besides, sub-grains with LAGBs also form via slip (Fig. 6d), which are not extensively seen in QS samples. LAGBs are closely related to local dislocation densities. The increase of strain rate is known to promote the pile-up of dislocations because dislocations do not get enough time to slip to and disappear at grain boundaries [16]. The increase of LAGBs with strain rate indicates a higher dislocation density in the dynamically deformed samples than in the QS deformed samples (Fig. 6e). DRX occurs around TBs due to the high stored lattice strain energy. Therefore, with increasing strain, secondary twinning starts to occur and the number of DRX grains increases. During plastic deformation, multiple nano-twinning events also occur within these sub-grains and DRX grains.

While experimental observation of the necking region revealed that deformation twinning, dislocation accumulation, grain refinement, and DRX all contributed to the strength and ductility of the material, deformation twinning was the dominant factor. The resulting TBs played a critical role in promoting dislocation accumulation, grain refinement and DRX. As significant grain refinement and DRX occurred around TBs mainly in the necking region at the late deformation stages, their overall contributions to the strength and ductility were relatively minor.

\section{Yield strength and flow stress}

At the yield point, all samples were of the same initial microstructure since the samples were mainly subjected to elastic tensile strain. Hence, the yield strength was primarily determined by the deformation conditions. Experimental results show that, while both increasing strain rate and reducing tem- perature increase yield strength, the strain rate affects yield strength more significantly than temperature does. High strain rate restricts the mobility of dislocations and gives less time for dislocation slip [34]. The flow stress also increases after yield because the increasing interactions of dislocations hinder their motion [13].

\section{Strain hardening at late deformation stages and $\sigma_{\mathrm{UTS}, \mathrm{T}}$}

The slopes of the true stress-strain curves in Fig. 1a after yielding are very similar, indicating similar strain hardening rates between QS and dynamic deformation. For dynamic deformation, the strain hardening rate and $\sigma_{\mathrm{UTS}, \mathrm{T}}$ are higher at cryogenic temperatures than at RT at the late stage of the true stress-true strain curves. It is well-known that low temperature promotes deformation twinning. TBs strengthen materials by effectively hindering the motion of dislocations [9,30,35]. Fig. 3 shows that LAGBs were located near TBs. A large amount of LAGBs is an indication of a high density of dislocations. As shown in Fig. 5, the dislocation densities near TBs were high. TBs intersect with each other and form a relatively stable network that contributes to the superior strength [26]. Fig. 3b, d present the intersections of some micron-sized twins, while Fig. $4 \mathrm{~b}$ shows the intersections of a few nano-sized twins. The $\sigma_{\mathrm{UTS}, \mathrm{T}}$ was higher and the strain hardening effect was more pronounced at cryogenic temperatures than at RT, which was ascribed to the increased twinning activities.

Grain refinement increases strength because GBs act as barriers to the motion of dislocations [36]. The increase in the density of LAGBs, as indicated by the increase in the number of sub-grains, also contributed to the strengthening, although they do not impede the dislocation motion as effectively as TBs and conventional high-angle GBs. Although DRX reduces the dislocation density that leads to softening [29], the resulting nanometer grain size strengthens materials through the Hall-Petch effect. Thus, the nett result of the combined effect of DRX could lead to hardening. The average grain size of the whole fracture area was finer at cryogenic temperatures than at RT, which is consistent with the observed higher fracture strength at cryogenic temperatures (Fig. 1a).

Increase in the density of TBs and decrease in the average grain size enhance the overall density of dislocations, which strengthens the alloy. Although increasing strain rate and reducing temperature only slightly increased the overall density of GNDs, they did change the spatial distribution of the GNDs. More even spread of dislocations was observed in samples dynamically deformed at cryogenic temperatures, which contributed to the yield and ultimate strengths of the material.

\section{Ductility}

Localised dislocation concentration has been condemned responsible for the poor ductility of materials at high strain rates [4]. These materials usually do not present significant deformation twinning activities. In contrast, materials that present increased ductility at high strain rates usually display significant twinning activities during high-strain-rate deformation [37,38]. Under this condition, full dislocation slip was relatively suppressed and deformation twinning was promoted. Deformation twinning contributes to the exceptional ductility at high strain rates through three mechanisms: (a) while TBs impede dislocation motion that strengthens alloys, they also increase the dislocation storage capability and consequently improve the 
strain hardening capability of materials, which delays necking and therefore improves ductility [39]; (b) TBs effectively prevent dislocation motion from one twin domain to another that significantly reduces localised dislocation concentration; and (c) TBs themselves facilitate deformation and improve ductility by allowing partial dislocation slip along the twin planes [26].

Meanwhile, the grain refinement that occurred during highstrain-rate deformation also contributed to the superior ductility. Two mechanisms operate for the grain refinement: (a) dislocation accumulation, interaction, tangling, and spatial rearrangement, and (b) DRX. While the first mechanism facilitates the movement of dislocations and reduces stress concentration, DRX effectively lowers the local dislocation density, which enables further plastic deformation and thus improves ductility at high strain rates. It is noted that deformation twinning and DRX both weaken the texture of the alloy in the neck area. Random crystallographic orientation relative to the loading direction facilitates the initiation of multiple slip systems and therefore contributes positively to the ductility [40].

\section{CONCLUSIONS}

We have successfully conducted high-strain-rate tensile deformation of bulk CrCoNi MEA until failure at RT, 200 and $77 \mathrm{~K}$ using an electromagnetic SHTB system and carried out extensive microstructural characterisation to understand the deformation mechanisms using EBSD/TKD and high-resolution TEM. The yield strength, $\sigma_{\mathrm{UTS}, \mathrm{T}}$ and the true strain at $\sigma_{\mathrm{UTS}, \mathrm{T}}$ of the alloy all increase with increasing strain rate and reducing temperature. The alloy exhibits the best dynamic mechanical properties ever reported at cryogenic temperatures. While the outstanding combination of strength and ductility at high strain rates is mainly attributed to the extensive deformation twinning, DRX and grain refinement, which occur around TBs in the necking region, also contribute to the strength and ductility at the late stages of the deformation process.

\section{Received 4 July 2021; accepted 15 September 2021;} published online 21 October 2021

1 Callister WD, Rethwisch DG. Materials Science and Engineering: An Introduction, the ninth ed., New York: John Wiley \& Sons Inc., 2006

2 Zhang Y, Zuo TT, Tang Z, et al. Microstructures and properties of high-entropy alloys. Prog Mater Sci, 2014, 61: 1-93

3 Gao X, Lu Y, Zhang B, et al. Microstructural origins of high strength and high ductility in an $\mathrm{AlCoCrFeNi}_{2.1}$ eutectic high-entropy alloy. Acta Mater, 2017, 141: 59-66

4 Hu X, Daehn GS. Effect of velocity on flow localization in tension. Acta Mater, 1996, 44: 1021-1033

5 Gludovatz B, Hohenwarter A, Thurston KVS, et al. Exceptional damage-tolerance of a medium-entropy alloy $\mathrm{CrCoNi}$ at cryogenic temperatures. Nat Commun, 2016, 7: 1-8

6 Gwalani B, Gorsse S, Choudhuri D, et al. Modifying transformation pathways in high entropy alloys or complex concentrated alloys via thermo-mechanical processing. Acta Mater, 2018, 153: 169-185

7 Liu J, Guo X, Lin Q, et al. Excellent ductility and serration feature of metastable $\mathrm{CoCrFeNi}$ high-entropy alloy at extremely low temperatures. Sci China Mater, 2019, 62: 853-863

8 Gludovatz B, Hohenwarter A, Catoor D, et al. A fracture-resistant highentropy alloy for cryogenic applications. Science, 2014, 345: 1153-1158

9 Christian JW, Mahajan S. Deformation twinning. Prog Mater Sci, 1995, 39: $1-157$

10 Zhao B, Huang $\mathrm{P}$, Zhang L, et al. Temperature effect on stacking fault energy and deformation mechanisms in titanium and titanium-aluminium alloy. Sci Rep, 2020, 10: 3086
11 Huang S, Li W, Lu S, et al. Temperature dependent stacking fault energy of FeCrCoNiMn high entropy alloy. Scripta Mater, 2015, 108: 44-47

12 Zhu YT, Liao XZ, Wu XL. Deformation twinning in nanocrystalline materials. Prog Mater Sci, 2012, 57: 1-62

13 Zhang TW, Ma SG, Zhao D, et al. Simultaneous enhancement of strength and ductility in a NiCoCrFe high-entropy alloy upon dynamic tension: Micromechanism and constitutive modeling. Int J Plast, 2020, 124: $226-246$

14 Guo Y, Du B, Liu H, et al. Electromagnetic Hopkinson bar: A powerful scientific instrument to study mechanical behavior of materials at high strain rates. Rev Sci Instruments, 2020, 91: 081501

15 Curtze S, Kuokkala VT. Dependence of tensile deformation behavior of TWIP steels on stacking fault energy, temperature and strain rate. Acta Mater, 2010, 58: 5129-5141

16 Wang L, Qiao JW, Ma SG, et al. Mechanical response and deformation behavior of $\mathrm{Al}_{0.6} \mathrm{CoCrFeNi}$ high-entropy alloys upon dynamic loading. Mater Sci Eng-A, 2018, 727: 208-213

17 Khan AS, Liu H. Variable strain rate sensitivity in an aluminum alloy: Response and constitutive modeling. Int J Plast, 2012, 36: 1-14

18 Khan AS, Yu S. Deformation induced anisotropic responses of Ti-6Al4V alloy. Part I: Experiments. Int J Plast, 2012, 38: 1-13

19 Gao P, Zhu SQ, An XH, et al. Effect of sample orientation and initial microstructures on the dynamic recrystallization of a magnesium alloy. Mater Sci Eng-A, 2017, 691: 150-154

20 Wang X, Huang C, Zou B, et al. Dynamic behavior and a modified Johnson-Cook constitutive model of Inconel 718 at high strain rate and elevated temperature. Mater Sci Eng-A, 2013, 580: 385-390

$21 \mathrm{Hu} \mathrm{M}$, Song W, Duan D, et al. Dynamic behavior and microstructure characterization of TaNbHfZrTi high-entropy alloy at a wide range of strain rates and temperatures. Int J Mech Sci, 2020, 182: 105738

22 Cao CM, Tong W, Bukhari SH, et al. Dynamic tensile deformation and microstructural evolution of $\mathrm{Al}_{x} \mathrm{CrMnFeCoNi}$ high-entropy alloys. Mater Sci Eng-A, 2019, 759: 648-654

23 Slone CE, Chakraborty S, Miao J, et al. Influence of deformation induced nanoscale twinning and FCC-HCP transformation on hardening and texture development in medium-entropy $\mathrm{CrCoNi}$ alloy. Acta Mater, 2018, 158: 38-52

24 Haase C, Barrales-Mora LA. Influence of deformation and annealing twinning on the microstructure and texture evolution of face-centered cubic high-entropy alloys. Acta Mater, 2018, 150: 88-103

25 Suwas S, Ray RK. Crystallographic Texture of Materials. London: Springer, 2014,123

26 Zhang Z, Sheng H, Wang Z, et al. Dislocation mechanisms and 3D twin architectures generate exceptional strength-ductility-toughness combination in CrCoNi medium-entropy alloy. Nat Commun, 2017, 8: 14390

27 Ma Y, Yuan F, Yang M, et al. Dynamic shear deformation of a CrCoNi medium-entropy alloy with heterogeneous grain structures. Acta Mater, 2018, 148: 407-418

28 Liao XZ, Huang JY, Zhu YT, et al. Nanostructures and deformation mechanisms in a cryogenically ball-milled Al-Mg alloy. Philos Mag, 2003, 83: 3065-3075

29 Zhu SQ, Yan HG, Liao XZ, et al. Mechanisms for enhanced plasticity in magnesium alloys. Acta Mater, 2015, 82: 344-355

30 Wang YB, Liao XZ, Zhao YH, et al. The role of stacking faults and twin boundaries in grain refinement of a $\mathrm{Cu}-\mathrm{Zn}$ alloy processed by highpressure torsion. Mater Sci Eng-A, 2010, 527: 4959-4966

31 Moussa C, Bernacki M, Besnard R, et al. About quantitative EBSD analysis of deformation and recovery substructures in pure tantalum. IOP Conf Ser-Mater Sci Eng, 2015, 89: 012038

32 Konijnenberg PJ, Zaefferer S, Raabe D. Assessment of geometrically necessary dislocation levels derived by 3D EBSD. Acta Mater, 2015, 99: 402-414

33 Sun JL, Trimby PW, Yan FK, et al. Shear banding in commercial pure titanium deformed by dynamic compression. Acta Mater, 2014, 79: 4758

34 Zheng Z, Balint DS, Dunne FPE. Rate sensitivity in discrete dislocation plasticity in hexagonal close-packed crystals. Acta Mater, 2016, 107: 1726 
35 Zhu YT, Liao XZ, Wu XL. Deformation twinning in bulk nanocrystalline metals: Experimental observations. JOM, 2008, 60: 60-64

36 Yang M, Yan D, Yuan F, et al. Dynamically reinforced heterogeneous grain structure prolongs ductility in a medium-entropy alloy with gigapascal yield strength. Proc Natl Acad Sci U S A, 2018, 115: 72247229

37 (Rusty) Gray III GT. High-strain-rate deformation: Mechanical behavior and deformation substructures induced. Annu Rev Mater Res, 2012, 42: 285-303

38 Wang $\mathrm{Y}$, Zhang Y, Godfrey A, et al. Cryogenic toughness in a low-cost austenitic steel. Commun Mater, 2021, 2: 44

39 Deng Y, Tasan CC, Pradeep KG, et al. Design of a twinning-induced plasticity high entropy alloy. Acta Mater, 2015, 94: 124-133

40 You ZS, Lu L, Lu K. Tensile behavior of columnar grained $\mathrm{Cu}$ with preferentially oriented nanoscale twins. Acta Mater, 2011, 59: 69276937

Acknowledgements The authors acknowledge the scientific and technical input and support from the Australian Microscopy and Microanalysis Research Facility node at the University of Sydney. Liao X was supported by the Australian Research Council (DP190102243).

Author contributions Liao X, Song M, and Li Y designed the study; Gao P prepared, acquired and analysed the microstructural data; Ma Z, Suo T, and Li Y conducted the mechanical tests; Gu J, Ni S, and Song M prepared the materials; Gao P, Liao X, and Mai YW wrote the paper. All authors discussed the results and contributed to the paper.

Conflict of interest The authors declare that they have no conflict of interest.

Supplementary information Supporting data are available in the online version of the paper.

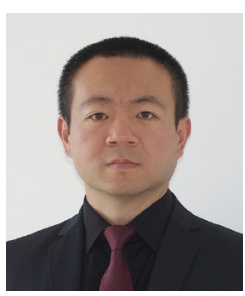

Peng Gao received his PhD degree in 2021 from the University of Sydney. He is employed as a research assistant at the School of Aerospace, Mechanical and Mechatronic Engineering, the University of Sydney. His research interest involves high/medium-entropy alloys, light alloys, microstructural characterisation and in-situ microscopy.

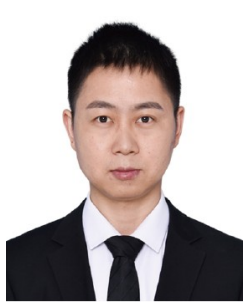

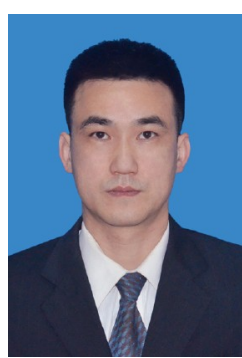

Min Song is a professor and vise dean of Powder Metallurgy Research Institute at Central South University. He serves as associate editor of Materials Characterization. He received his $\mathrm{PhD}$ degree in 2005 at Dartmouth College, USA. His current research interests involve the deformation mechanisms of metallic materials, including: metals and alloys, bulk nanocrystalline materials, HEAs and metal matrix composites.

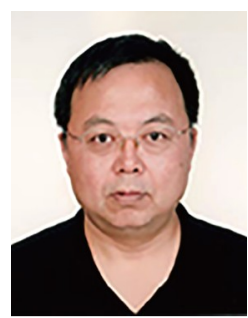

Yulong Li is a professor of Civil Aviation School and a chair professor at the School of Aeronautics, NPU. He received his $\mathrm{PhD}$ degree in solid mechanics from NPU in 1992. His research interests include dynamic response and failure of structures under impact loading, constitutive relationship for materials, experimental techniques for high-strain-rate behaviors of materials, as well as, numerical simulation of materials and structures under impact.

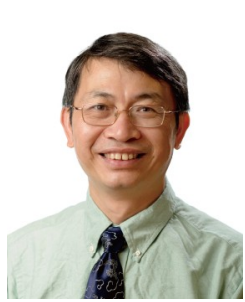

Xiaozhou Liao received his $\mathrm{PhD}$ degree in physics from the University of Sydney. He is currently a professor at the School of Aerospace, Mechanical and Mechatronic Engineering, the University of Sydney. He uses advanced electron microscopy to examine materials at the atomic scale, laying the groundwork for the design of superior materials for a wide range of applications.

\section{CrCoNi中熵合金优异的高应变速率拉伸力学性能}

高鹏 $^{1 \dagger}$, 马自豪 ${ }^{3 \dagger}$, 顾及 ${ }^{2}$, 倪颂 $^{2}$, 索涛 ${ }^{3}$, 李玉龙 ${ }^{*}$, 宋旼 ${ }^{2 *}$, 米耀荣 ${ }^{1}$, 廖晓舟 ${ }^{1 *}$

Zihao Ma is currently pursuing his $\mathrm{PhD}$ degree at the School of Aeronautics, Northwestern Polytechnic University (NPU). He is studying the mechanical behavior and microstructure evolution of high-entropy alloys under dynamic impact.

\begin{abstract}
摘要 拥有优异的力学强度和拉伸塑性对于结构合金是非常重要的. 然而, 合金在高应变速率或者低温下变形时通常拉伸塑性非常有限. 本 文展示了块体 $\mathrm{CrCoNi}$ 中商合金在高应变速率和低温下优异的综合拉 伸力学性能. 我们使用一种带冷却装置的电磁霍普金森拉杆装置得到 了在 $2000 \mathrm{~s}^{-1}$ 和 $77 \mathrm{~K}$ 下完整的拉伸力学曲线. 结果表明合金在 $1.8 \mathrm{GPa}$ 下 具有超高的拉伸真应力 $\left(\sigma_{\mathrm{UTS}, \mathrm{T}}\right)$, 在 $\sigma_{\mathrm{UTS}, \mathrm{T}}$ 下的拉伸真应变为 $54 \%$. 该合 金优异的力学性能主要归因于大量变形孪晶的启动. 高应变速率和低 温都促使了孪晶的形成. 变形孪晶、滑移、动态再结晶引起的晶粒细 化促进了加工硬化, 从而增加了拉伸塑性.
\end{abstract}

